\title{
A Pulsed Method for Measuring the Conductivity on Solid Ionic Conductors
}

\author{
J. Certo,* J. M. Perdigão, C. S. Furtado and A. J. Ferreira
}

ICEMS, Departamento de Engenharia Electrotécnica, Universidade de Coimbra, 3030 Coimbra, Portugal

\section{Abstract}

Electrode polarization is the main drawback when measuring the conductivity on solid ionic conductors. To overcome this inconvenience, a twoterminal pulsed method was used to measure, under strong electrode polarization, the dc conductivity on Na- $\beta$-alumina samples. The results agree well with those obtained with impedance spectroscopy. (C) 1999 Elsevier Science Limited. All rights reserved

\section{Introduction}

The ionic conductivity (or resistivity) is the most important parameter to characterize a solid ionic conductor. In this type of materials, electrode polarization is the main drawback when electric measurements have to be made. ${ }^{1}$

There are some methods to deal with polarization, ${ }^{2}$ the most used of which is the impedance spectroscopy. However, the results obtained by this technique are, in many cases, not too precise. This is the case when the $\mathrm{Z}$ or $\mathrm{Y}$ plots include distorted arcs. ${ }^{3}$ Another method to measure conductivity under polarization effects is the pulsed method proposed by Johnson et al. ${ }^{2}$ The polarization is avoided by choosing a short pulse duration.

In this paper a pulsed method to measure the ionic conductivity is presented. The measurements were done under electrode polarization. The method was tested both in an equivalent electric circuit of a polycrystalline $\mathrm{Na}-\beta$-alumina sample and in a real sample.

*To whom correspondence should be addressed. Fax: + 35139-7-6247; E-mail: certo@alumni.dee.uc.pt

\section{Experimental Arrangement}

The pulsed method presented here is a two-terminal technique. The experimental arrangement is illustrated in Fig. 1. The circuit is fed by a pulse generator (10 Mhz band). The sample is a solid electrolyte with two silver electrodes. $R_{p}$ is a precision metal-film resistor (tolerance $0.1 \%$, temperature coefficient $15 \mathrm{ppm}$ ) used to evaluate the current intensity, $i(t)$, across the sample. $D$ is a low leakage, low capacitance diode (JPAD5), the function of which is to avoid the sample-discharge over the low output impedance of the pulse generator. The $100 \mathrm{M} \Omega$ resistor regulates the discharge between pulses. The pulse has a frequency of about $10 \mathrm{~Hz}$ and a width which depends on the impedance characteristics of the sample. Its regulation will be discussed later on. The measurement set is a two channel digital real-time oscilloscope (Tektronix TDS 360) in association with two DI-FET high speed precision operational amplifiers (OPA $602 \mathrm{AP}$ ) mounted as voltage followers used to increase the input impedances of the scope to $10^{12} \Omega$.

\section{Method Description and Experimental Results}

Pellets of $\mathrm{Na}-\beta$-alumina were fabricated from powders with compositions of $9.69 \mathrm{wt} \% \mathrm{Na}_{2} \mathrm{O}$ and $90.31 \mathrm{wt} \% \mathrm{Al}_{2} \mathrm{O}_{3}$. The specimens used in the measurements, all made at room temperature $\left(\approx 25^{\circ} \mathrm{C}\right)$, had an aspect ratio $(1 / A)$ equal to $2 \mathrm{~cm}^{-1}$ $\left(1 \approx 0.7 \mathrm{~cm}, A \approx 0.6 \times 0.6 \mathrm{~cm}^{2}\right)$. Ionically blocking electrodes were applied by firing silver paste on polished surfaces.

To get the impedance equivalent circuit of one of the pellets, plots of $\mathrm{Z}$ and $\mathrm{Y}$ were displayed in an impedance analyser (Hewlett Packard 4194A). According to the a.c. data obtained, the equivalent circuit which best fits the results is represented in Fig. 2, with $R_{b}=500 \Omega, R_{g b}=5 \mathrm{~K} \Omega, C_{g b}=330 \mathrm{pF}$, and $C_{d l}=39 \mathrm{nF}$. 
The method was first tested by replacing the sample of the scheme of Fig. 1 by the equivalent lumped circuit just described. The precision resistor $R_{p}$ has a value of $10 \mathrm{~K} \Omega$. In a first attempt, to separate the effects of $C_{g b}$ and $C_{d l}$ on the response of the circuit, $C_{d l}$ was removed [Fig. 3(a)]. The corresponding scope display is shown in Fig. 3(b). If, during a pulse, $C_{g b}$ gets full charged $\left[v_{2}(t)\right.$ tends to a horizontal straight line], then no current flows through it. Thus, the current $i(t)$ in $R_{p}$ is the same in $R_{b}$ and $R_{g b}$. In this case, at the end of a pulse $\left(t=0^{-}: i\left(0^{-}\right)=I, v_{1}\left(0^{-}\right)=V_{1}\right.$ and $\left.v_{2}\left(0^{-}\right)=V_{2}\right)$, the total resistance of the circuit $\left(R_{t}=R_{b}+R_{g b}\right.$ $=5.5 \mathrm{~K} \Omega)$ is obtained as $R_{t}=V_{2} / I=V_{2} R_{p} /$ $\left(V_{1}-V_{2}\right)$, where $V_{1}-V_{2}$ is the voltage drop across $R_{p}, V_{2}$ is the voltage across $R_{t}$ and $I$ is the current in $R_{p}$ and $R_{t}$. In the case of Fig. 3, for a pulse width of $50 \mu \mathrm{s}, R_{t}=5.46 \mathrm{~K} \Omega$, which gives an error of $0.7 \%$. Attention must be paid in such a way that pulse width is large enough to let $C_{g b}$ be fully charged.

A second attempt is done by using the complete equivalent circuit [Fig. 4(a)]. The corresponding $v_{1}(t)$ and $v_{2}(t)$ displays are shown in Fig. 4(b). At the end of each pulse, the voltage drop across $R_{t}$ is $V_{2}-V_{3}$, where $V_{3}$ is the $C_{d l}$ voltage. As the voltage at the end of charging $\left(t=0^{-}\right)$is the same at the

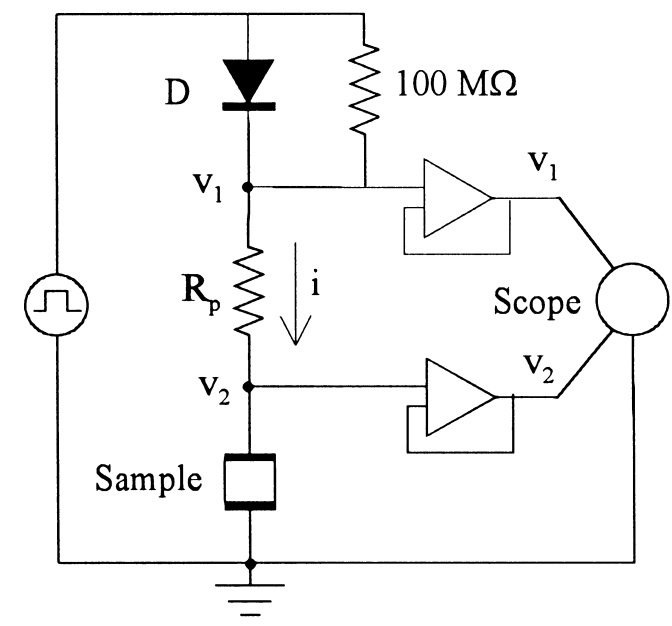

Fig. 1. Schematic diagram of the pulsed conductivity measurement circuit.

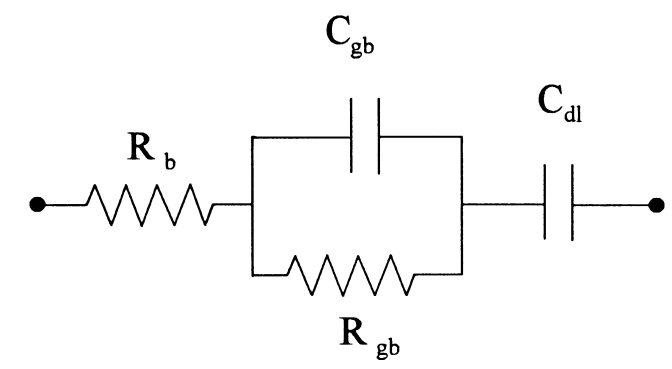

Fig. 2. Equivalent circuit of a sodium $\beta$-alumina pellet used. $R_{b}=$ intragranular (bulk) resistance. $R_{g b}$ and $C_{g b}=$ grainboundary resistance and capacitance. $C_{d l}=$ double layer capacitance of electrode/electrolyte interface. ${ }^{4}$ beginning of discharging $(t=0), V_{3}$ can be determined from the $v_{2}(t)$ decay curve of Fig. 4(b). Thus, $V_{3}$ is given by the intersection of the extrapolated line in the $v_{2}(t)$ decay plot, representing the $C_{d l}$ voltage, with the $v$ axis. On the other hand, at $t=0^{-}$, if the capacitor $C_{g b}$ is already charged and the capacitor $C_{d l}$ is still being charged $\left(C_{g b}<<C_{d l}\right)$, the current $i(t)$ flowing through $C_{d l}$ is the same in $R_{p}, R_{b}$ and $R_{g b}$. So, the total resistance of the equivalent circuit of a $\beta$-alumina sample is $\left(V_{2}-V_{3}\right) / I$ and can be calculated from

$$
R_{t}=\frac{V_{2}-V_{3}}{V_{1}-V_{2}} R_{p}
$$

a)

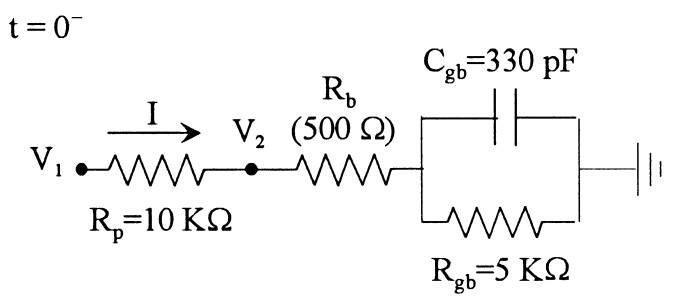

b)

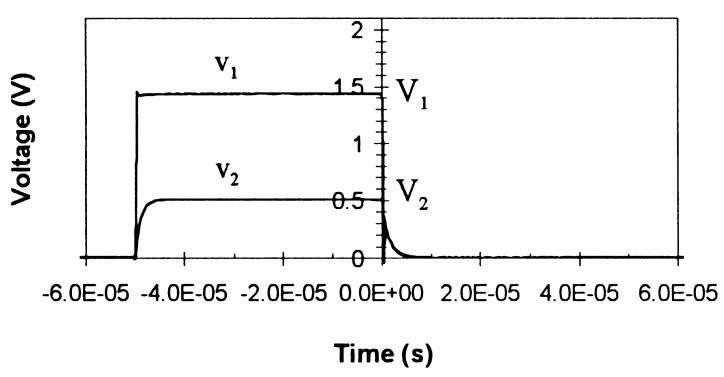

Fig. 3. (a) Precision resistor and equivalent circuit without $C_{d l}$. (b) Scope display when the sample of Fig. 1 is replaced by this equivalent circuit.

a) $t^{=} 0^{-}$

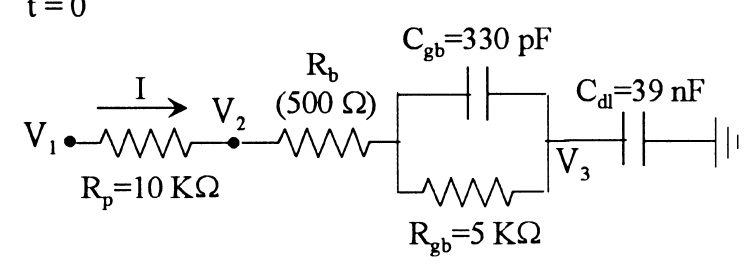

b)

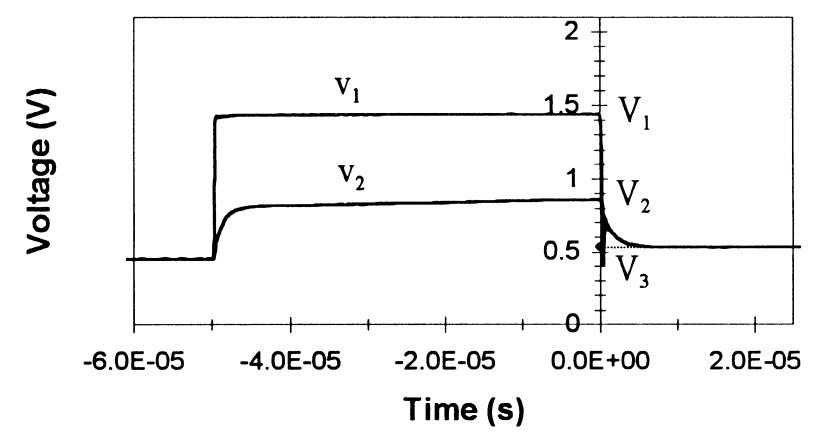

Fig. 4. (a) Precision resistor and the complete equivalent circuit. (b) Scope display when the sample of Fig. 1 is replaced by this equivalent circuit. 


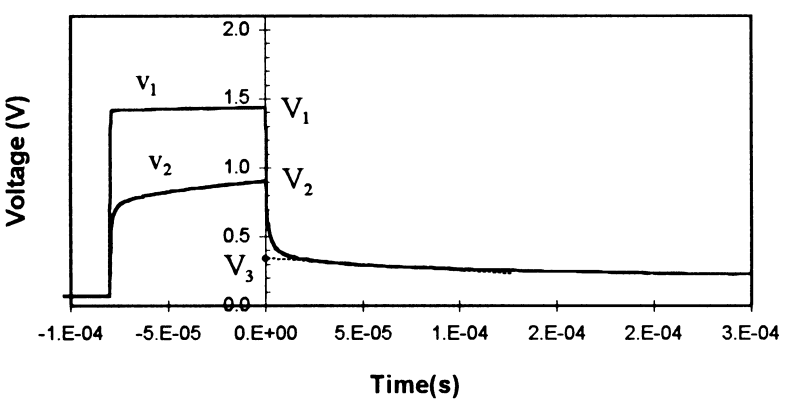

Fig. 5. Scope display when the sample of Fig. 1 is a $\beta$-alumina specimen.

In the case of Fig. $4, R_{t}$ is equal to $5.6 \mathrm{~K} \Omega$, which gives an error of $1.8 \%$. Finally, the pulsed method was tested directly in a pellet of $\mathrm{Na}-\beta$-alumina. The total resistance got from complex impedance spectroscopy was about $9.3 \mathrm{~K} \Omega$. The $v_{1}(t)$ and $v_{2}(t)$ displays are presented in Fig. 5, with a pulse width equal to $80 \mu \mathrm{s}$. As the last part of the $v_{2}(t)$ decay curve, which represents the voltage across $C_{d l}$, is not a straight line, its intersection with the $v$ axis cannot be precisely found. To overcome this inconvenience a tangent line to the $v_{2}(t)$ decay curve is plotted in such a way that its direction follows the trend of the last part of the variation of $v_{2}(t)$, as represented in Fig. 5. Then the values of $V_{1}, V_{2}$, and $V_{3}$ can be easily read from this figure. Using eqn (1), $R_{t}$ can be calculated. From this calculation the Na- $\beta$-alumina sample measured has $R_{t}=10.4 \mathrm{~K} \Omega$, which deviates $12 \%$ from the corresponding value obtained by impedance spectroscopy.

\section{Conclusions}

The pulsed method presented in this paper aims to overcome the difficulties in measuring d.c. ionic conductivity on 'two-electrodes' samples due to polarization. This technique offers the following advantages: it is not affected by polarization, shows good precision, and requires equipment not too expensive. Obviously only the total ionic conductivity can be easily measured. The results agree well with those obtained with impedance spectroscopy.

\section{References}

1. Kinser, D. L. and Hench, L. L., J. Am. Ceram. Soc., 1969, 52(12), 638-647.

2. Johnson Jr, R. T., Biefield, R. M., Knotek, M. L. and Morosin, B., J. Electrochem. Soc., 1976, 123(5), 680-687.

3. Macdonald, J. R., Impedance Spectroscopy. Emphasizing Solid Materials and Systems. John Wiley \& Sons, New York, 1987.

4. Hooper, A., J. Phys. D: Appl. Phys., 1977, 10, 1487-1496. 